\title{
Identification Scheme for Fractional Hammerstein Models With the Delayed Haar Wavelet
}

\author{
Kajal Kothari, Utkal Mehta, Vineet Prasad, and Jito Vanualailai
}

\begin{abstract}
The parameter identification of a nonlinear Hammerstein-type process is likely to be complex and challenging due to the existence of significant nonlinearity at the input side. In this paper, a new parameter identification strategy for a block-oriented Hammerstein process is proposed using the Haar wavelet operational matrix (HWOM). To determine all the parameters in the Hammerstein model, a special input excitation is utilized to separate the identification problem of the linear subsystem from the complete nonlinear process. During the first test period, a simple step response data is utilized to estimate the linear subsystem dynamics. Then, the overall system response to sinusoidal input is used to estimate nonlinearity in the process. A single-pole fractional order transfer function with time delay is used to model the linear subsystem. In order to reduce the mathematical complexity resulting from the fractional derivatives of signals, a HWOM based algebraic approach is developed. The proposed method is proven to be simple and robust in the presence of measurement noises. The numerical study illustrates the efficiency of the proposed modeling technique through four different nonlinear processes and results are compared with existing methods.
\end{abstract}

Index Terms-Fractional-order models, Haar wavelet, Hammerstein model, nonlinear process, operational matrix, time delay.

\section{INTRODUCTION}

I $\mathrm{N}$ industrial applications, various advanced processes are inherently nonlinear. The processes with negligible nonlinearity can be treated as linear to reduce complexity; however significant nonlinearity can not be ignored. Several engineering problems are studied widely using two block-oriented models, namely the Hammerstein model and Wiener model. Identification of these models have been an important topic for a long time. The Hammerstein model can represent various nonlinear systems accurately, e.g., fuel cells, chemical processes, magneto-rheological dampers, supercapacitor systems, battery, DC/DC boost converters, aerodynamic models, and biological processes [1], [2]. This highlights the import-

Manuscript received January 10, 2020; revised February 7, 2020; accepted February 22, 2020. Recommended by Associate Editor Wei He. (Corresponding author: Utkal Mehta.)

Citation: K. Kothari, U. Mehta, V. Prasad, and J. Vanualailai, "Identification scheme for fractional Hammerstein models with the delayed Haar wavelet," IEEE/CAA J. Autom. Sinica, vol. 7, no. 0, pp. 1-10, Feb. 2020.

K. Kothari, U. Mehta, and V. Prasad are with the School of Engineering and Physics, The University of the South Pacific, Laucala campus, Fiji (email: s11151029@student.usp.ac.fj; utkal.mehta@usp.ac.fj; s11122160@student.usp.ac.fj).

J. Vanualailai is with the School of Computing, Information and Mathematical Sciences, The University of the South Pacific, Laucala campus, Fiji (email: jito.vanualailai@usp.ac.fj).

Color versions of one or more of the figures in this paper are available online at http://ieeexplore.ieee.org.

Digital Object Identifier 10.1109/JAS.2020.1003093 ance of the Hammerstein model and encourages researchers to explore and develop better techniques.

In the Hammerstein model, the input to the linear subsystem is altered by the nonlinearity. Therefore, nonlinearity significantly affects the dynamics of the actual system. It is noteworthy, that nonlinearity elevates computational overhead during identification along with the overall cost, as one has to estimate not only the linear subsystem but also the nonlinearity. Naturally, this leads to the issue of identifying the entire nonlinear model efficiently with minimal computational effort.

In the literature, various techniques have been reported on nonlinear process identification using integer-order (classical) models. The Hammerstein process was estimated using the special test signal in [3] and further extended for HammersteinWiener processes in [4], [5]. The separate block-oriented noniterative relay feedback (SRF) method was illustrated in [6]. Mehta and Majhi [7] presented the non-iterative relay feedback (NRF) method to determine the structure prior to the parameters of the Hammerstein model. The Hammerstein system with time delay has been accurately identified using a recursive least squares method in [8]. Recently, a separate block-oriented parameter identification method for Hammerstein systems using least squares was described in [9]. Furthermore, another special input based identification of Hammerstein-Wiener nonlinear system with noise was discussed in [10]. Even though some efficient integer-order techniques have been developed so far, a fractional domain approach is yet to be fully explored for nonlinear process identification.

Initially, Aoun et al. [11] introduced fractional calculus based identification techniques for Hammerstein models. The interconnected complex nonlinear processes and their identification problem were addressed in [12]. A fractionalorder model for a thermal system was illustrated in [13] for large variations in temperature. A novel frequency domain approach was developed and presented in [14] whereby Wiener and Hammerstein dynamics were depicted in terms of poles and zeros of the estimated linear approximation, which produced favorable results. However, it was solved with an iterative minimization algorithm which could be caught in local minima. This work was revisited and extended by Giordano and Sjöberg using a time-domain approach to avoid the local minima problem by [15]. Allafi et al. [16] demonstrated identification of the Hammerstein-Wiener model based on a simplified refined instrumental variable method with some prior knowledge. The operational matrix approach using the fractional Taylor basis was developed and 
utilized for a numerical solution of linear and nonlinear fractional differential equations in [17]. Zhao et al. [2] presented a parametric identification technique of commensurate fractional-order quasi-linear kinetic battery model (KiBaM). Parameter identification methods of fractional-order chaotic system with time delay was described in [18] whereby an artificial bee colony algorithm was used to solve the multi-dimensional optimization problem. A modified Volterra least mean square (LMS) algorithm for fractional Hammerstein modeling was suggested in [19]. A modified artificial bee colony algorithm was proposed for nonlinear fractional system parameter estimation in [20]. Fractional-order multi-input single-output Hammerstein process identification was well illustrated in [21] which utilized the well known genetic algorithm with recursive least squares. Fractional-order stochastic gradient algorithm was developed and discussed for Hammerstein-type ARMAX system identification in [22]. The state-space model approach for fractional Hammerstein process was described in [23]. Neural network based nonlinear fractional process modeling verified in real time with wind turbines was depicted in [24]. Recently, Wang et al. [1] presented a fully parametric identification (FPI) technique using two algorithms for a fractional Hammerstein process. This FPI method accurately estimates all the parameters of a commensurate model, however, it can not estimate delay. Another technique for Hammerstein process identification using the LMS algorithm and spline interpolation was presented in [25]. A Haar wavelet based parameter estimation technique for fractional nonlinear processes was proposed in [26]. However, this method was not developed for block oriented processes. Furthermore, linear subsystems were considered without time delay.

All the aforementioned fractional approaches do not separate nonlinearity and linear subsystems. The merits of separate identification of linear and nonlinear subsystems make it attractive for researchers. One can have freedom of selecting any one of the available identification techniques for a linear block. Moreover, it is possible to select a non-iterative method for nonlinearity estimation and can improve accuracy due to less number of unknowns to handle at a time. These key points have motivated authors to develop a systematic approach using fractional calculus theory.

In this paper, a parameter identification strategy is presented for a block-oriented Hammerstein-type nonlinear process where the linear part is a continuous-time fractional-order process with time delay. A special test signal constituting unit step and sinusoidal signals, has been applied to the entire nonlinear process for the purpose of identification. Firstly, the unit step response is utilized for the estimation of linear subsystem parameters. It is observed that the step input activates nonlinearity in the form of amplitude changes. In fact, a step response can directly be used for linear subsystem identification without loss of generality. Subsequently, a sinusoidal excitation is utilized to estimate the nonlinear and linear subsystem static gain. Additionally, the HWOM omits the direct calculation of fractional derivatives and transforms complex expressions into a simple algebraic matrix multiplications. This strategy is aimed at precise identification with reduced complexity. The Riemann-Liouville definition (see Appendix) is utilized in this work to handle the fractional derivatives.

The outline of this paper is as follows: Section II introduces the Hammerstein model and Haar wavelet for fractional-order integration. The proposed technique to identify parameters in the linear part and subsequently, nonlinearities is discussed in Section III. Section IV focuses on four numerical examples for performance evaluation and some concluding remarks are provided in Section V.

\section{HAMMERSTAIN MODELS AND HAAR WAVELET}

A Hammerstein-type process is represented using blockoriented models, whereby static nonlinearity is succeeded by a dynamic linear subsystem in a cascaded manner as shown in Fig. 1. The input $u(t)$ and output $y(t)$ are the measurable quantities whereas the intermediate signal $\hat{v}(t)$ is an experimentally immeasurable quantity. It is assumed that $G(s)$ is a stable process and the static nonlinear function $f(u)$ is monotonic and crossing at the origin. Let the linear subsystem $G(s)$ be represented by familiar a fractional-order single-pole model as

$$
G(s)=\frac{Y(s)}{V(s)}=\frac{b_{0}}{a_{1} s^{\alpha}+a_{0}} e^{-\theta s}
$$

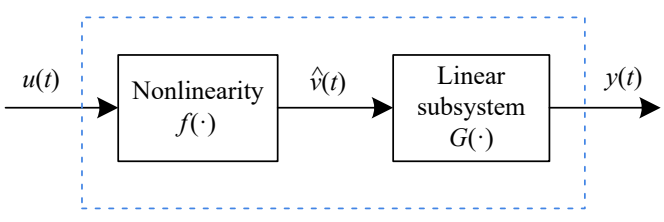

Fig. 1. Hammerstein-type nonlinear process model.

where coefficients $\left(a_{0}, a_{1}, b_{0} \in \mathbb{R}\right)$, fractional-order $\left(\alpha \in \mathbb{R}^{+}\right)$, and $\theta$ represents the input time delay. The purpose of any identification technique is to represent actual system dynamics with more accurate models. The low-order model of any system is highly desirable especially when modeling with a fractional approach. This is due to memory constraints when implementing fractional-orders in real time. Furthermore, it is well-known that many practical systems in the industry are of low-order dynamics, whereby higher-order complicated models are reduced to lower-order compact forms. The advantage of working with a lower-order compact model is the reduction in complexity and computational overhead for identification procedures and the ease in implementing model-based controller design.

Various types of static nonlinearities can be approximated easily using the polynomial form [7]. In this work, for identification purposes, a nonlinear polynomial function is considered up to the fourth order, which is sufficient to represent static memoryless nonlinearities efficiently with reduced complexity. Therefore, the nonlinear block can be illustrated as follows:

$$
\hat{v}(t)=f(u(t))=\sum_{i=1}^{4} c_{i} u^{i}(t) .
$$


However, one can increase the order of the polynomial if higher accuracy is demanded.

\section{A. Concept of Fractional-Order Integration for Delayed Haar Wavelet}

In the proposed method, orthogonal Haar wavelet functions are utilized due to high precision, mathematical simplicity, noise immunity and ease of implementation with other standard algorithms. Computationally, Haar wavelets are faster compared to other functions of the wavelet family and can be defined as [27]

$$
h_{m}(t)=h_{1}\left(2^{i} t-k T_{f}\right)
$$

where $T_{f}$ is the total time period, $m=2^{i}+k$, whereby $i(i \geq 0)$ and $k\left(0 \leq k \leq 2^{i}\right)$ are integers

$$
h_{0}(t)=1 \text {, for } 0 \leq t<T_{f} \text {, and } h_{1}(t)= \begin{cases}1 & 0 \leq t<\frac{T_{f}}{2} \\ -1 & \frac{T_{f}}{2} \leq t<T_{f} \\ 0 & \text { otherwise. }\end{cases}
$$

Now, an arbitrary function $x(t) \in L^{2}\left[0, T_{f}\right]$ can be written in terms of Haar wavelets for first $M$ number of terms as

$$
x(t)=\sum_{i=0}^{M-1} x_{i} h_{i}(t)=X_{M}^{T} H_{M}(t) .
$$

Here, $X_{M}=\left[x_{0}, x_{1}, \ldots, x_{M-1}\right]^{T}$ is the Haar coefficient vector and $H_{M}(t) \triangleq\left[h_{0}(t), h_{1}(t), \ldots, h_{M-1}(t)\right]^{T}$ is the Haar function vector. If collocation points are considered as $t_{i}=(2 i-1) T_{f} / 2 M, i=1,2, . ., M$, the $M$-square Haar matrix $\Phi_{M \times M}$ can be defined by

$$
\Phi_{M \times M}=\left[H_{M}\left(\frac{1}{2 M} T_{f}\right) H_{M}\left(\frac{3}{2 M} T_{f}\right) \ldots H_{M}\left(\frac{2 M-1}{2 M} T_{f}\right)\right] .
$$

The integration of Haar wavelet can be obtained by its multiplication with square matrix $P_{M \times M}^{\alpha}$. The algebraic expression of fractional-order integration (FOI) of Haar wavelet is written as

$$
\left(\mathcal{I}^{\alpha} H_{M}\right)(t) \approx P_{M \times M}^{\alpha} H_{M}(t)
$$

where $M$-square matrix $P_{M \times M}^{\alpha}$ is called the HWOM of FOI which is computed as given in [27]

$$
P_{M \times M}^{\alpha}=\Phi_{M \times M} F^{\alpha} \Phi_{M \times M}^{-1}
$$

where $F^{\alpha}$ is the $M$-square generalized operational matrix of FOI for block pulse functions [28] and can be described as

$$
F^{\alpha}=\left(\frac{T_{f}}{M}\right)^{\alpha} \frac{1}{\Gamma(\alpha+2)}\left(\begin{array}{ccccc}
f_{1} & f_{2} & f_{3} & \ldots & f_{M} \\
0 & f_{1} & f_{2} & \ldots & f_{M-1} \\
\vdots & \ddots & f_{1} & \ldots & f_{M-2} \\
\vdots & & \ddots & \ddots & \vdots \\
0 & \ldots & \ldots & 0 & f_{1}
\end{array}\right)
$$

where $f_{1}=1, f_{q}=q^{\alpha+1}-2(q-1)^{\alpha+1}+(q-2)^{\alpha+1}$ and $q=2,3, \ldots$, $M$. Similarly, in the case of delayed input, the considered delayed Haar wavelet function $H_{M}(t-\theta)$ can be characterized as

$$
H_{M}(t-\theta)=Z H_{M}(t)
$$

where $\theta$ is the input time delay and $Z$ is the Haar wavelet operational matrix of delay which can be written as given in [29],

$$
Z=\Phi_{M \times M} E \Phi_{M \times M}^{-1}
$$

where $M$-square matrix $E$ is the generalized delay operational matrix [28]

$$
E_{M \times M}=\left[\begin{array}{ccccccc}
0 & \cdots & 0 & 1 & 0 & \cdots & 0 \\
0 & \cdots & 0 & 0 & 1 & \cdots & 0 \\
\vdots & & \vdots & \vdots & \vdots & \ddots & \vdots \\
0 & \cdots & 0 & 0 & 0 & \cdots & 1 \\
0 & \cdots & 0 & 0 & 0 & \cdots & 0 \\
\vdots & & \vdots & \vdots & \vdots & & \vdots \\
0 & \cdots & 0 & 0 & 0 & \cdots & 0
\end{array}\right] .
$$

Therefore, performing fractional integration of delayed Haar function $H_{M}(t-\theta)$ using (6) and (9) results in,

$$
\left(\mathcal{I}^{\alpha} H_{M}\right)(t-\theta) \approx Z\left(\mathcal{I}^{\alpha} H_{M}\right)(t)=Z P_{M \times M}^{\alpha} H_{M}(t) .
$$

Thus, $\left(\mathcal{I}^{\alpha} H_{M}\right)(t-\theta)$ is thereby simply obtained by matrix multiplication of $H_{M}(t)$ with $P_{M \times M}^{\alpha}$ and $Z$ matrices.

In the presented work, the fractional calculus and operational matrix based method [29] developed for singleinput single-output process is incorporated and extended to the approximate Hammerstein model.

\section{IDENTIFICATION TECHNIQUE FOR HAMMERSTEIN MODELS}

Complete identification is accomplished using a special test signal in two steps. Fig. 5 shows an example of a special test input signal and its response. It is noted that the input and output satisfies the condition of $u(t) \in L^{2}\left[0, T_{f}\right]$ and $y(t) \in L^{2}\left[0, T_{f}\right]$. Furthermore, the input signal $u_{\text {step }}$ is persistently exciting for the linear subsystem and $u_{\text {sine }}$ is persistently exciting for overall nonlinear process. First, the unit step input response is utilized for estimation of linear subsystem parameters. Subsequently, the sine wave input response is utilized for parameters of unknown nonlinearity in

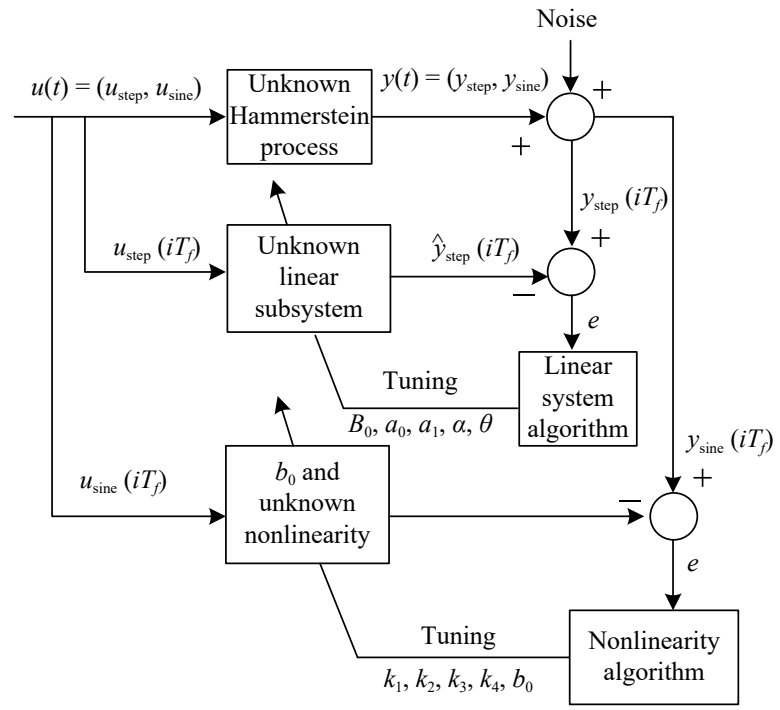

Fig. 2. Comprehensive nonlinear process identification scheme. 


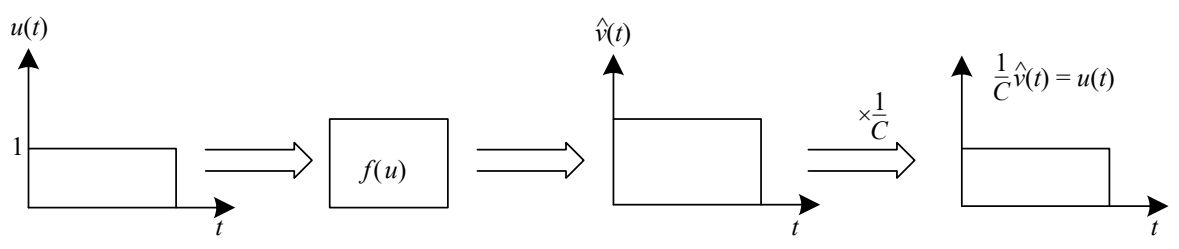

Fig. 3. Utilization of unit step response for linear subsystem without loss of generality.

the second step. The proposed strategy for identification is depicted in Fig. 2.

\section{A. Identification of the Linear Subsystem}

For Hammerstein-type processes, nonlinearity is activated in the form of an amplitude change for constant input. Moreover, when unit step input is applied, the amplitude of a nonlinear block's output is a multiple of the nonlinearities gain. In (2), when constant unity input is applied, total nonlinear gain would be $C=\sum_{i=1}^{4} c_{i}$. Therefore, the intermediate output $\hat{v}(t)=C u(t)$ shown in Fig. 3 .

Therefore, the substitution of $V(s)=C U(S)$ in (1) yields

$$
G(s)=\frac{Y(s)}{C U(s)}=\frac{b_{0}}{a_{1} s^{\alpha}+a_{0}} e^{-\theta s} .
$$

Let, $C b_{0}=B_{0}$, therefore

$$
\frac{Y(s)}{U(s)}=\frac{C b_{0}}{a_{1} s^{\alpha}+a_{0}} e^{-\theta s}=\frac{B_{0}}{a_{1} s^{\alpha}+a_{0}} e^{-\theta s} .
$$

Equation (14) can be simplified as

$$
a_{1} s^{\alpha} Y(s)+a_{0} Y(s)=B_{0} U(s) e^{-\theta s} .
$$

Expressing (15) in the time-domain yields

$$
a_{1} \mathcal{D}^{\alpha} y(t)+a_{0} y(t)=B_{0} u(t-\theta) .
$$

Integrating both sides with order $\alpha_{n}$ gives

$$
a_{1} y(t)+a_{0} \mathcal{I}^{\alpha} y(t)=B_{0} \mathcal{I}^{\alpha} u(t-\theta) .
$$

Measured data can now be expressed in terms of the Haar wavelet as

$$
\begin{gathered}
u(t-\theta)=U^{T} H_{M}(t-\theta) \\
y(t)=Y^{T} H_{M}(t)
\end{gathered}
$$

where $Y^{T}=\left[y_{1}, y_{2}, \ldots, y_{M}\right], U^{T}=\left[u_{1}, u_{2}, \ldots, u_{M}\right]$ and superscript $T$ denotes the transpose. Substituting (18) and (19), (17) can be rewritten as

$$
a_{1} Y^{T} H_{M}(t)+a_{0} Y^{T} \mathcal{I}^{\alpha} H_{M}(t)=B_{0} U^{T} \mathcal{I}^{\alpha} H_{M}(t-\theta) .
$$

Replacing the fractional integral and time delay by operational matrices yields

$$
a_{1} Y^{T} H_{M}(t)+a_{0} Y^{T} P_{M \times M}^{\alpha} H_{M}(t)=B_{0} U^{T} Z P_{M \times M}^{\alpha} H_{M}(t) .
$$

Hence

$$
Y^{T}\left(a_{1} I+a_{0} P_{M \times M}^{\alpha}\right)=U^{T} Z\left(B_{0} P_{M \times M}^{\alpha}\right)
$$

where $I$ denotes the $M$-square identity matrix.

$$
Y^{T}=U^{T} Z\left(B_{0} P_{M \times M}^{\alpha}\right)\left(a_{1} I+a_{0} P_{M \times M}^{\alpha}\right)^{-1} .
$$

Note that an inverse matrix of $\left(a_{1} I+a_{0} P_{M \times M}^{\alpha}\right)$ exists because the determinant of the identity matrix, as well as orthogonal $P_{M \times M}^{\alpha}$ matrix, is always non-zero. Moreover, coefficients $\left(a_{0}, a_{1} \in R^{+}\right)$ensure that the matrix $\left(a_{1} I+a_{0} P_{M \times M}^{\alpha}\right)$ is reversible. Finally, the output expression can be obtained using (19) and (23) as

$$
y(t)=U^{T} Z\left(B_{0} P_{M \times M}^{\alpha}\right)\left(a_{1} I+a_{0} P_{M \times M}^{\alpha}\right)^{-1} H_{M}(t) .
$$

Now, using an optimization approach one can estimate the unknown parameters, $a_{0}, a_{1}, \alpha, \theta$ and $B_{0}=C b_{0}$. It is to be noted that $b_{0}$ is multiplied with nonlinear gain $C$, therefore an independent $b_{0}$ needs to be obtained in the second step with nonlinearity parameters $c_{i}(i=1,2,3,4)$.

Remark 1: The model in (1) represents linear fractional first order model which is a very basic model and generally preferred. However, one can use any order model with a greater number of poles and zeros. Consider the generalized $n$ pole, $m$-zero model as given below

$$
\frac{Y(s)}{U(s)}=\frac{\left(b_{m} s^{\beta_{m}}+b_{m-1} s^{\beta_{m-1}}+\cdots+b_{0} s^{\beta_{0}}\right)}{\left(a_{n} s^{\alpha_{n}}+a_{n-1} s^{\alpha_{n-1}}+\cdots+a_{0} s^{\alpha_{0}}\right)} e^{-\theta s}
$$

where $a_{i}(i=0, \ldots, n), \quad b_{j}(j=0, \ldots, m) \quad$ are constants; $\alpha_{i}(i=0, \ldots, n), \beta_{j}(j=0, \ldots, m)$ are arbitrary real numbers; and $\theta$ represents the time delay. The final output expression $y(t)$ for the aforementioned transfer function can be written as below.

$$
y(t)=U^{T} Z N D^{-1} H_{M}(t)
$$

where $\quad D=\left(a_{n} I+a_{n-1} P_{M \times M}^{\alpha_{n}-\alpha_{n-1}}+\cdots+a_{0} P_{M \times M}^{\alpha_{n}-\alpha_{0}}\right) \quad$ and $N=\left(b_{m} P_{M \times M}^{\alpha_{n}-\beta_{m}}+b_{m-1} P_{M \times M}^{\alpha_{n}-\beta_{m-1}}+\cdots+b_{0} P_{M \times M}^{\alpha_{n}-\beta_{0}}\right)$. The results can be enhanced as per the desired accuracy using the aforementioned output expression. Hence, the method is flexible with the generalized transfer function. However, this can increase the implementation cost and computational efforts.

\section{B. Identification of the Nonlinearity}

The identification of the nonlinear block is accomplished using a sinusoidal response. As mentioned earlier

$$
\hat{v}(t)=f(u(t))=c_{1} u(t)+c_{2} u(t)^{2}+c_{3} u(t)^{3}+c_{4} u(t)^{4} .
$$

If $u(t)$ and $\hat{v}(t)$ are expressed in terms of Haar wavelets, then

$$
\begin{aligned}
\hat{v}(t) & =V^{T} H_{M}(t) \\
u(t) & =U^{T} H_{M}(t) .
\end{aligned}
$$

Substituting (28) into (27) results in,

$$
\begin{aligned}
\hat{v}(t)= & c_{1} U^{T} H_{M}(t)+c_{2}\left[U^{T} H_{M}(t)\right]^{2} \\
& +c_{3}\left[U^{T} H_{M}(t)\right]^{3}+c_{4}\left[U^{T} H_{M}(t)\right]^{4} .
\end{aligned}
$$

The Haar wavelets are piecewise constant and can be 
expanded in the form of block pulse functions as,

$$
H_{M}(t)=\Phi_{M \times M} \psi_{M}(t)
$$

where the vector $\psi_{M}(t)$ represents block pulse functions. Using (31), the nonlinear terms of expression (30) can be simplified as given in the literature [26]

$$
\begin{aligned}
{\left[U^{T} H_{M}(t)\right]^{2} } & =\left[U^{T} \Phi_{M \times M} \psi_{M}(t)\right]^{2} \\
& =U^{T} \Phi_{M \times M} \psi_{M}(t)\left[\psi_{M}(t)\right]^{T}\left[\Phi_{M \times M}\right]^{T} U .
\end{aligned}
$$

Let

$$
U^{T} \Phi_{M \times M}=\left[\lambda_{1} \lambda_{2} \lambda_{3} \ldots \lambda_{M}\right]=[\lambda] .
$$

Using the definition of block pulse functions, one can write

$$
\psi_{M}(t)\left[\psi_{M}(t)\right]^{T}=\left[\begin{array}{cccc}
\psi_{1}(t) & & & 0 \\
& \psi_{2}(t) & & \\
& & \ddots & \\
0 & & & \psi_{M}(t)
\end{array}\right]
$$

and substituting the values of (33) and (34), (32) can be further simplified as

$$
\left[U^{T} H_{M}(t)\right]^{2}=[\lambda]\left[\begin{array}{cccc}
\psi_{1}(t) & & & 0 \\
& \psi_{2}(t) & & \\
& & \ddots & \\
0 & & & \psi_{M}(t)
\end{array}\right][\lambda]^{T}
$$

which finally yields

$$
\left[U^{T} H_{M}(t)\right]^{2}=\left[\lambda_{1}^{2} \lambda_{2}^{2} \ldots \lambda_{M}^{2}\right] \psi_{M}(t)=\left[\lambda^{2}\right] \psi_{M}(t) .
$$

Similarly, one can obtain

$$
\begin{aligned}
& {\left[U^{T} H_{M}(t)\right]^{3}=\left[\lambda_{1}^{3} \lambda_{2}^{3} \ldots \lambda_{M}^{3}\right] \psi_{M}(t)=\left[\lambda^{3}\right] \psi_{M}(t) .} \\
& {\left[U^{T} H_{M}(t)\right]^{4}=\left[\lambda_{1}^{4} \lambda_{2}^{4} \ldots \lambda_{M}^{4}\right] \psi_{M}(t)=\left[\lambda^{4}\right] \psi_{M}(t) .}
\end{aligned}
$$

Now, rewriting all the terms in (30) using (33), (36)-(38) results in

$$
\hat{v}(t)=c_{1}[\lambda] \psi_{M}(t)+c_{2}\left[\lambda^{2}\right] \psi_{M}(t)+\left[\lambda^{3}\right] \psi_{M}(t)+\left[\lambda^{4}\right] \psi_{M}(t) .
$$

Furthermore, using (24) one can derive the expression of the linear subsystem output for sinusoidal excitation in terms of intermediate simulated input $\hat{v}(t)$

$$
y(t)=V^{T} Z\left(b_{0} P_{M \times M}^{\alpha}\right)\left(a_{1} I_{M \times M}+a_{0} P^{\alpha}\right)^{-1} H_{M}(t) .
$$

In the aforementioned expression, $b_{0}$ is unknown, therefore it can be replaced by the $b_{0}=B_{0} / C$. Note that $B_{0}$ is a known parameter from the previous step. Therefore, $b_{0}$ and $V^{T}$ can be replaced using (39), and (40) can now be rewritten as

$$
\begin{aligned}
& y(t)=\left(c_{1}[\lambda]+c_{2}\left[\lambda^{2}\right]+c_{3}\left[\lambda^{3}\right]+c_{4}\left[\lambda^{4}\right]\right) \psi_{M}(t) \\
& {\left[H_{M}(t)\right]^{-1} \frac{B_{0}}{C} Z\left(P_{M \times M}^{\alpha}\right)\left(a_{1} I+a_{0} P_{M \times M}^{\alpha}\right)^{-1} H_{M}(t) .}
\end{aligned}
$$

This expression of output $y(t)$ is used to find $c_{i}(i=1,2,3,4)$, keeping previously estimated linear subsystem parameters constant. An independent $b_{0}$ is obtained using $b_{0}=B_{0} / C$ from estimated $c_{i}$ values.
The proposed approach is summarized into the following simple steps.

Step 1: Generate a special input signal, constituting a unit step and sinusoidal signal, for excitation of the Hammerstein process.

Step 2: Record the measurable input and output data $u(t)$ and $y(t)$ for $2 M$ samples.

Step 3: Identify the linear subsystem parameters $a_{0}, a_{1}, \alpha, \theta$ and also $B_{0}$ from $M$ samples (here, a half-time test data is basically recorded for a step input).

Step 4: Calculate $b_{0}$ and $c_{i}$ from the remaining half-time $(M)$ samples.

The time-moment weighted integral performance criteria, for identification of both linear and nonlinear subsystems, is used as an objective function to minimize identification error. The integral of squared-time-weighted-error (ISTE) is used to estimate the system parameters and can be written in accordance to estimated values

$$
I S T E=\min _{\rho} \sum_{k=1}^{M}\left[k\left(y_{\text {data }}(k)-y(k)\right)\right]^{2}
$$

where $\rho$ is the vector of unknown parameters, $y(k)$ and $y_{\text {data }}(k)$ are the simulated response and collected actual data respectively, and $M$ denotes the total number of samples. The objective of the optimization is to find model parameters that would ideally reduce the ISTE to minimum value. The MATLAB function fsolve is used to compute the best-estimated parameters which would satisfy the objective function in (42). The function solve has three different algorithms: trust-regiondogleg, trust-region, and Levenberg-Marquardt. In this work, the Levenberg-Marquardt algorithm was utilized and its detailed convergence analysis is depicted in [30]. Moreover, the persistence of excitation is a sufficient condition for parameter convergence [31], and as mentioned earlier, inputs for the proposed method are persistently exciting. Furthermore, owing to the convex nature and feasible solution of problem (42), the convergence of the prevailing mathematical optimization algorithms is always guaranteed. However, it is always important to assume the appropriate initial value of parameters. More appropriate initial guesses make convergence occur more quickly with more accuracy. There is no mathematical formula or theory available for the selection of initial guesses. The proposed identification method gave satisfactory results in the finite trials. The first optimization procedure was performed, and the result was used as the new initial guess after which the succeeding trial is performed and so on [28]. The selection of initial guesses affects the convergence and number of trials required for the optimization procedure.

\section{NUMERICAL STUDY}

The proposed identification scheme for fractional-order Hammerstein models, was implemented and verified in MATLAB. Several numerical examples, studied in the literature in [1], [6], [7], and [11], were simulated to evaluate the performance with a significant nonlinearity. The optimum number of samples is considered on the basis of the acceptable accuracy of identification. The performance was verified in the following examples with respect to number of samples, 
different input types, and measurement noises.

Example 1: Consider a fractional-order Hammerstein process $p_{1}$ studied in [11] whereby the linear subsystem is $G_{1}(s)=e^{-s} / 1+2 s^{0.5}$ and nonlinearity is $f_{1}(u)=u(t)+2 u^{2}(t)$. The importance of nonlinear process identification becomes apparent when analyzing the function characteristic of a nonlinear element with the $3 \mathrm{D}$ plot as shown in Fig. 4 . The input compounded of step and sinusoidal signals for the process is illustrated in Fig. 5.

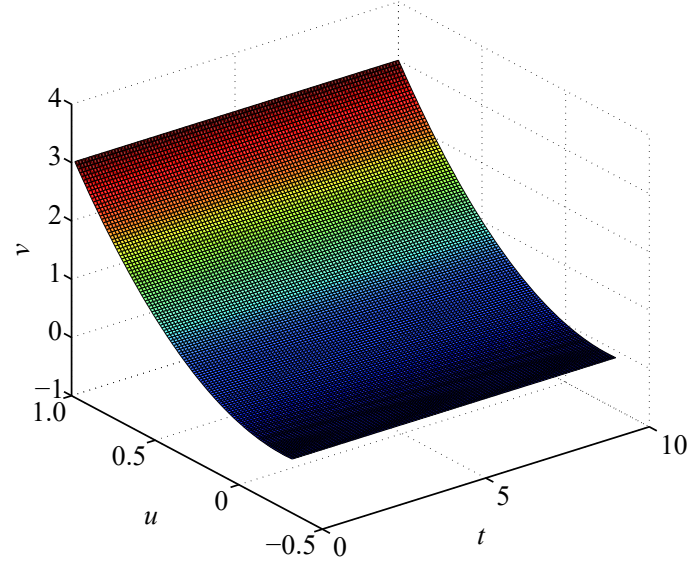

Fig. 4. Physical interpretation of nonlinearity via $3 \mathrm{D}$ plot for $f_{1}(u)$.
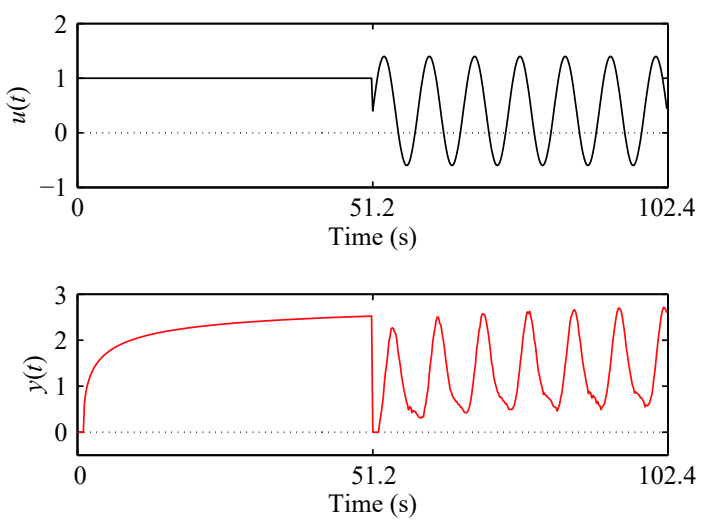

Fig. 5. Input output data for identification.

As outlined in the previous section, a unit step response $u(t)=C \hat{v}(t)$ was considered without loss of generality and after that, the linear subsystem parameters, $B_{0}, a_{0}, a_{1}, \alpha$ and $\theta$ were estimated from the measured output. Using the second part of measured data from the sinusoidal response, the nonlinearity polynomial coefficients, $c_{1}, c_{2}, c_{3}, c_{4}$, and independent linear subsystem gain $b_{0}$, were obtained. Table I shows the approximated linear subsystems, nonlinearity and time-domain errors for studied examples. Figs. 6 and 7 compare the actual and estimated linear and nonlinear parameters.

Noise is a key factor in determining the effectiveness of the technique for any real process identification. The identification algorithm should be robust in order to process real-time measurement values. The proposed method was verified under measurement noises with $\mathrm{SNR}=10 \mathrm{~dB}$ and

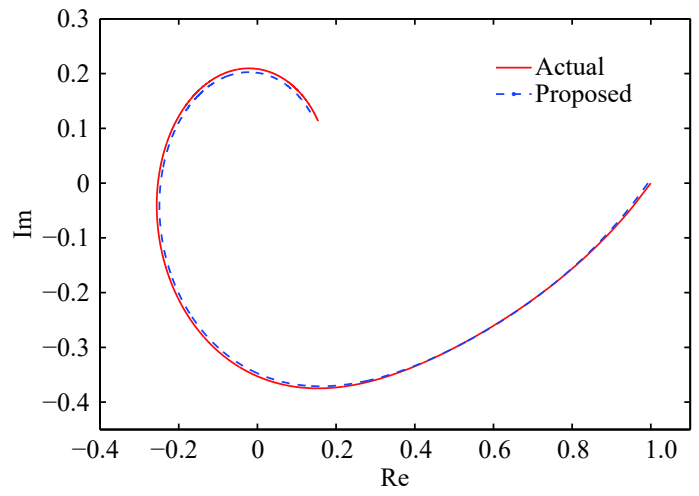

Fig. 6. Frequency response Nyquist plot for $G_{1}(j \omega)$.

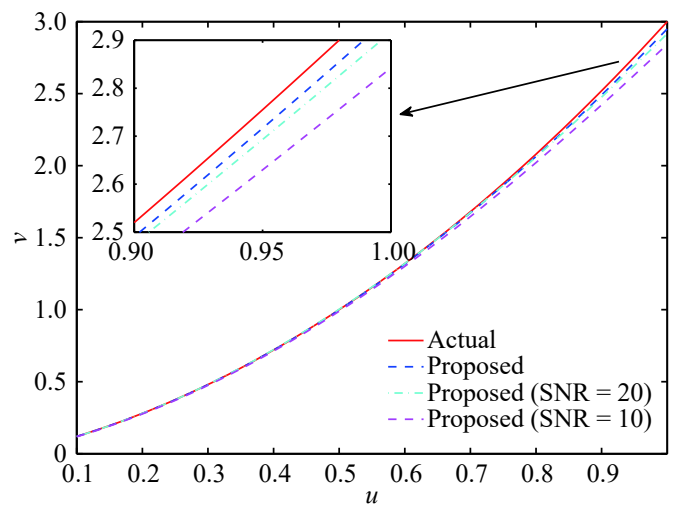

Fig. 7. Identified nonlinearity for Example-1.

$\mathrm{SNR}=20 \mathrm{~dB}$. The results plotted in Fig. 8 depict robustness when the measured data is contaminated by noise. This result can be credited to the noise immunity property of the Haar wavelets.

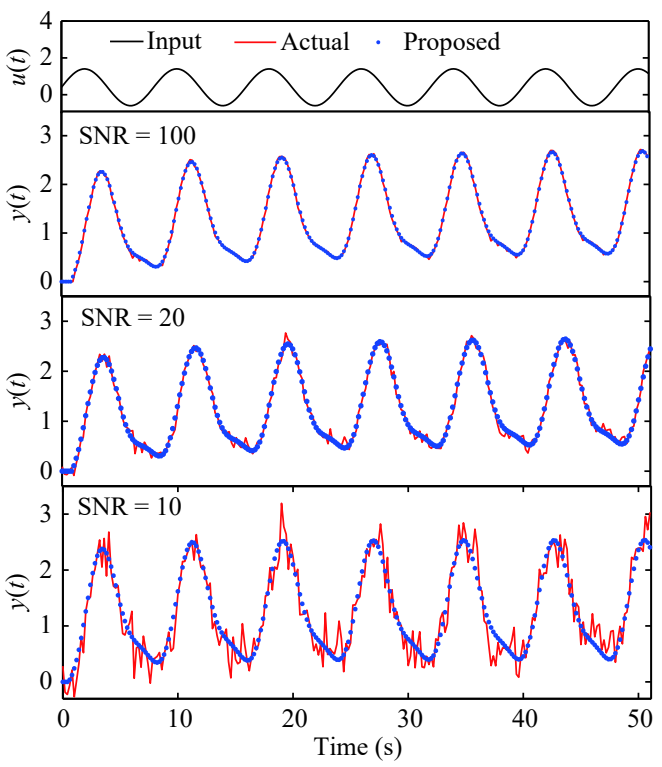

Fig. 8. Results with noisy output signals for example-1.

The datalength is another important factor to determine the trade-off between the accuracy and speed of the identification. 
TABLE I

RESULTS AND COMPARISONS

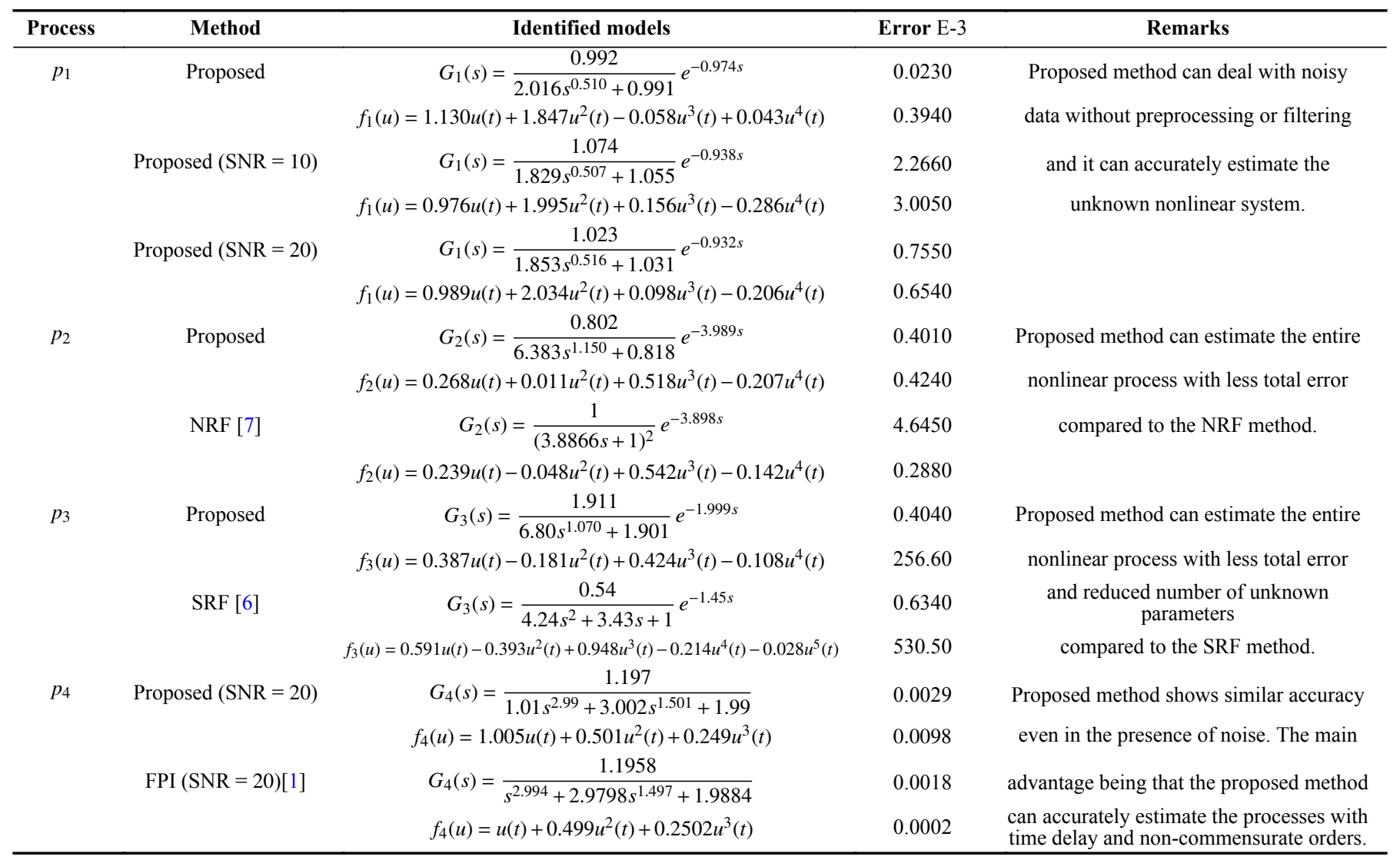

$\mathrm{NRF}:=$ non-iterative relay feedback [7]; SRF:= separate non-iterative relay feedback [6]; FPI:= fully parametric identification [1].

Furthermore, the complexity in separately identifying the error introduced is profound due to the operational matrix approximation. Nonetheless, the error due to approximation is dependent on the datalength $M$. It is obvious that a small number of samples accelerates the identification but can result in incorrect estimation. Table II shows the various datalengths with time-domain errors. It clearly indicates the effect of the datalength on the precision of the identification. Overall, it is observed that the Haar wavelet based method works favorably with sample points $M=256$.

TABLE II

EFFECT OF Datalength ON ACCURACy OF IDENTIFICATION

\begin{tabular}{cccc}
\hline Datalength & $E_{L S}$ & $E_{N L}$ & $E_{\text {total }}$ \\
\hline$M=512$ & $1.91 E-4$ & $4.18 E-4$ & $6.09 E-4$ \\
$M=256$ & $1.87 E-4$ & $4.91 E-4$ & $6.78 E-4$ \\
$M=128$ & $2.05 E-4$ & $8.22 E-4$ & $10.27 E-4$ \\
$M=64$ & $2.64 E-2$ & $20.0 E-2$ & $22.64 E-2$ \\
\hline
\end{tabular}

$E_{L S}:=$ error with linear model; $E_{N L}:=$ error with nonlinear model; $E_{\text {total }}=$ total error for estimation.

In order to verify identification results with other deterministic or random signals, the same process $p_{1}$ is identified with various input types. It is noteworthy that the estimation procedure was considered with the same initial guess, samples, and number of iterations. The resulting timedomain errors are listed in Table III. It is seen that the presented technique is almost consistent with any input test signal. Furthermore, the normalized mean values of all parameters are depicted in Fig. 9 for various inputs.

Example 2: In this example, a higher-order Hammerstein process $p_{2}$ studied in [7] is considered. The linear subsystem is given by $G_{2}(s)=\frac{1}{(s+1)^{2}+(2 s+1)^{3}} e^{-s}$ and the input

TABLE III

VARIOUS INPUTS FOR $b_{0}$ AND NONLINEARITY IDENTIFICATION

\begin{tabular}{cccc}
\hline Input type & $E_{L S}$ & $E_{N L}$ & $E_{\text {total }}$ \\
\hline Sine & $1.82 E-4$ & $3.94 E-4$ & $5.76 E-4$ \\
Multi-sine & $8.03 E-4$ & $43.4 E-4$ & $51.4 E-4$ \\
Sawtooth & $2.12 E-4$ & $3.00 E-4$ & $5.12 E-4$ \\
Multi-step & $1.06 E-4$ & $4.44 E-4$ & $5.51 E-4$ \\
Random & $3.05 E-4$ & $22.0 E-4$ & $25.5 E-4$ \\
\hline
\end{tabular}

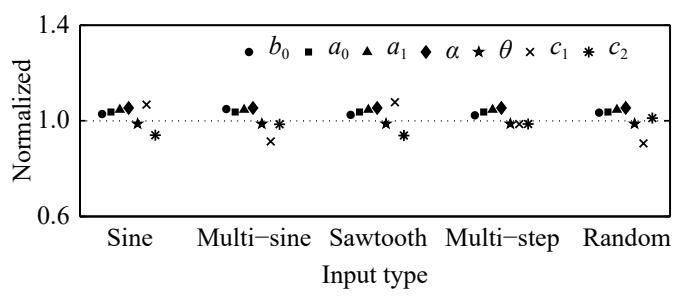

Fig. 9. Effect of input types on parameter identification for $G_{1}(s)$. 
nonlinearity is $f_{2}(u)=1.5\left(1-e^{-0.5 u}\right)|u|$. The estimated linear and nonlinear subsystems are given in Table I. The comparisons of the linear and nonlinear subsystems with the NRF method [7] are shown in Figs. 10 and 11. It can be seen that the proposed method is accurate for both subsystems. The complete estimated Hammerstein model response is compared with actual process in Fig. 12.

Example 3: The nonlinear process $p_{3}$ was considered from [6], with linear subsystem $G_{3}(s)=e^{-s} /(s+1)^{5}$ and input

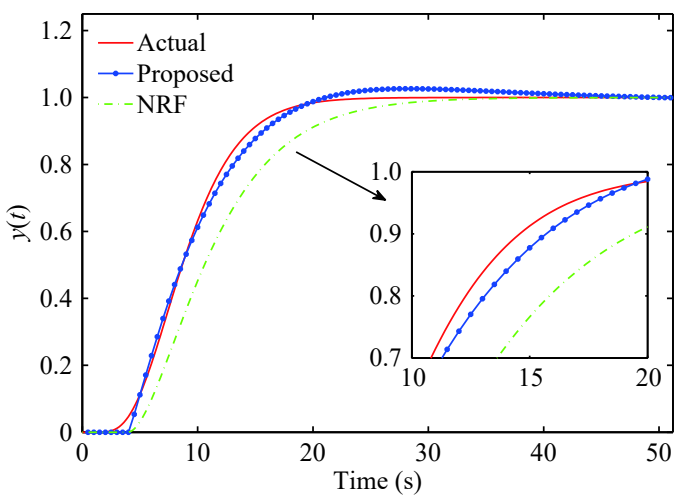

Fig. 10. Comparison of step responses for linear subsystem $G_{2}(s)$.

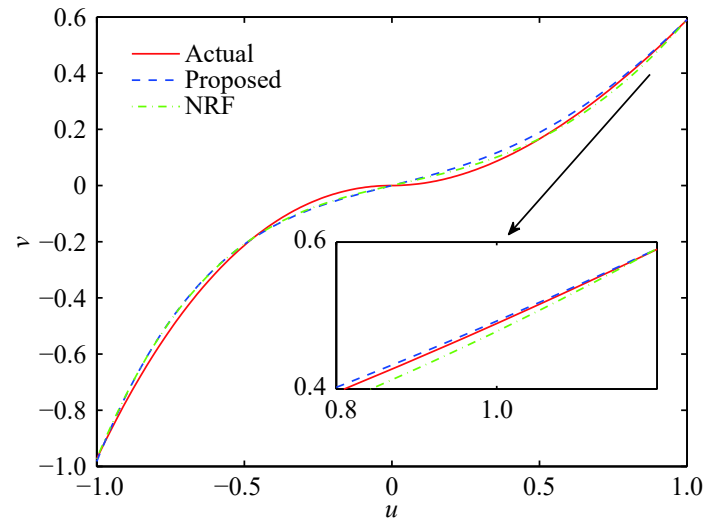

Fig. 11. Comparison of static nonlinearities $f_{2}(u)$.
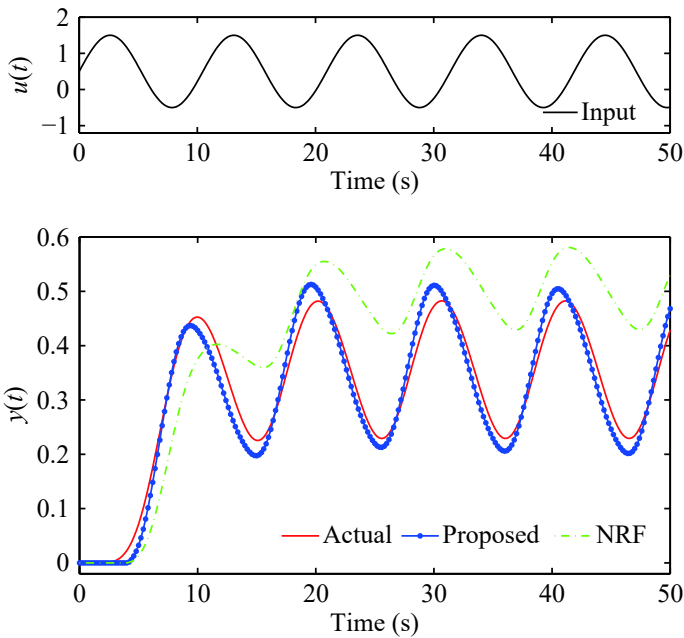

Fig. 12. Comparison of sinusoidal responses for process $p_{2}$. nonlinearity $f_{3}(u)=\left(1-e^{-0.75 u}\right)|u|$. Using the proposed method, the identification results are shown in Table I. The identification errors and the comparative plots in Figs. 13 and 14 clearly demonstrate the superiority of the proposed method over the SRF [6] method.
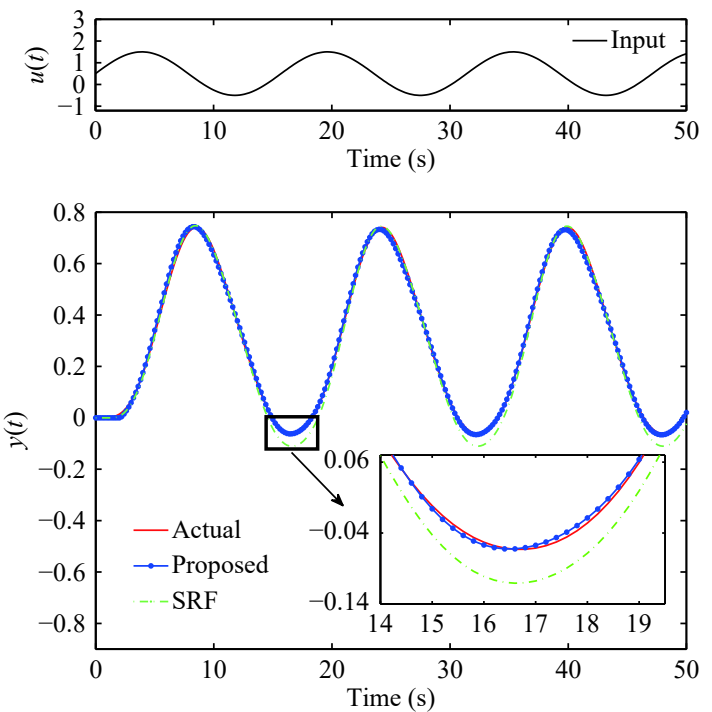

Fig. 13. Comparison of sinusoidal responses for process $p_{3}$.

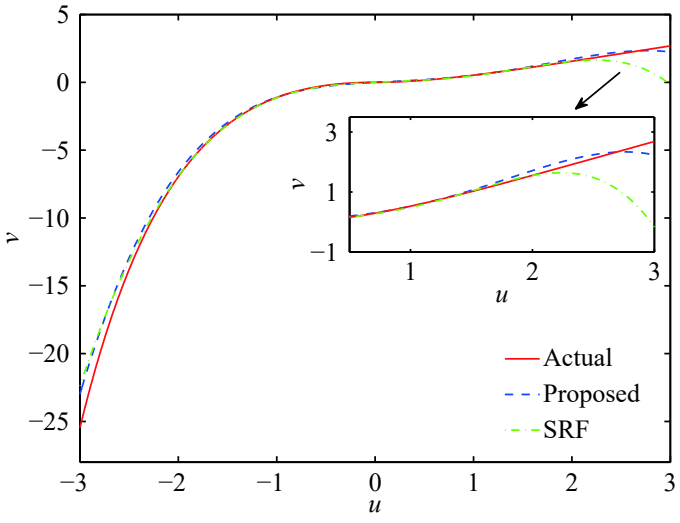

Fig. 14. Comparison of static nonlinearities $f_{3}(u)$.

Example 4: The nonlinear process $p_{4}$ was considered from the recent literature [1], with linear subsystem $G_{4}(s)=$ $1.2 / s^{3}+3 s^{1.5}+2$ and input nonlinearity $f_{4}(u)=u+0.5 u^{2}+$ $0.25 u^{3}$. In order to make a fair comparison, the linear and nonlinear subsystems were estimated with the same number of unknown parameters as in [1]. The proposed method has been enhanced as given in Remark 1 for comparison purposes. The identified linear and nonlinear subsystems with measurement noise $\mathrm{SNR}=20 \mathrm{~dB}$ are shown in Table I. The identification errors demonstrate similar accuracy. The main advantage of the proposed method over the FPI method [1] is that the proposed method can accurately estimate the system with time delay and non-commensurate orders without prior knowledge.

The convergence analysis for linear and nonlinear subsystems are depicted in Figs. 15 16, respectively. The convergence of objective function ISTE can be achieved 


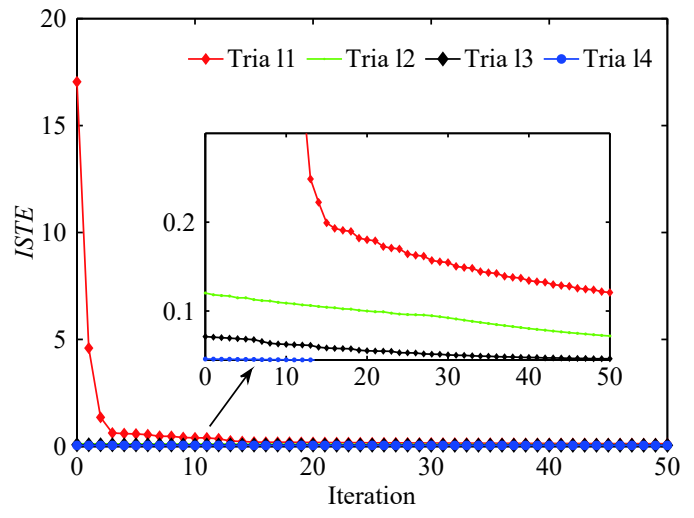

Fig. 15. Convergence analysis of linear subsystem using step response.

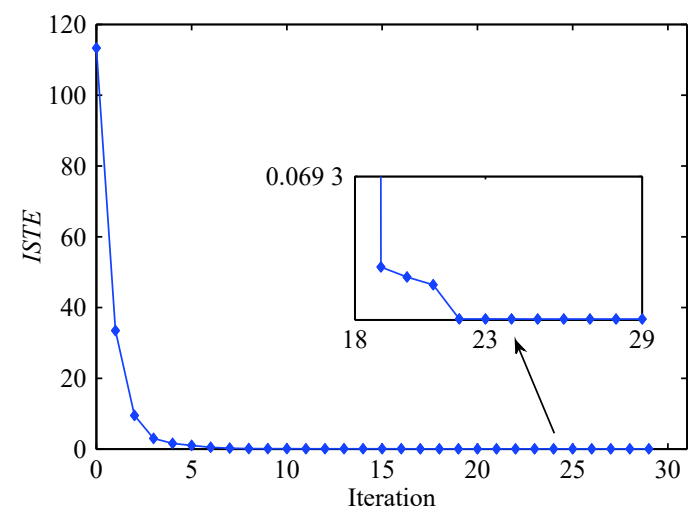

Fig. 16. Convergence analysis of nonlinear subsystem using sine response.

towards a minimum possible value in a finite number of trials. The first optimization procedure was performed and the result was used as the new initial guess after which the succeeding trial was performed and so on. It is obvious that for each trial, ISTE value decreases as iteration increases. Finally, the optimum values of parameters were obtained with minimum ISTE as shown in Fig. 15.

\section{CONCLusions}

As seen from the results presented, the Haar wavelet-based identification strategy for a class of the Hammerstein model definitely reduces the complexity with fractional-order estimation. No prior information is needed and it simultaneously identifies the delay parameter with other linear subsystem parameters. It is noteworthy that the presented approach completely separates the linear and nonlinear subsystem identifications. The proposed technique permits the freedom to select any of the available fractional identification techniques for linear models. Moreover, it is possible to select a non-iterative method for a nonlinearity estimation. It is observed from the results that the scheme is robust with noisy signal data, eliminating additional denoising steps. Both linear and nonlinear subsystems are accurately identified using a simple HWOM based algebraic approach. Furthermore, the approach can be enhanced with a higher-order fractional model in order to achieve more accuracy but requires more computational efforts. It would be interesting to further extend this work for the Wiener or Hammerstein-Wiener models.

\section{APPENDIX \\ FRACTIONAL CALCULUS}

Fractional calculus is a generalization of non-integer (real) order integration and differentiation and its operator is generally defined as

$$
{ }_{a} D_{t}^{\alpha}= \begin{cases}\frac{\mathrm{d}^{\alpha}}{\mathrm{d} t^{\alpha}} & \alpha>0 \\ 1 & \alpha=1 ; \\ \int_{a}^{t}(\mathrm{~d} \tau)^{-\alpha} & \alpha<0\end{cases}
$$

where $a$ and $t$ are the bounds of the operation and $\alpha(\alpha \in \mathbb{R})$ is the order of operation.

There exist numerous definitions to characterize fractional integration and differentiation. In the proposed work, the Riemann-Liouville definition is utilized which can be written as

$$
{ }_{a} D_{t}^{\alpha} f(t)=\frac{1}{\Gamma(n-\alpha)}\left(\frac{\mathrm{d}}{\mathrm{dt}}\right)^{n} \int_{a}^{t} \frac{f(\tau)}{(t-\tau)^{\alpha+1-n}} \mathrm{~d} \tau
$$

where $n-1<\alpha<n, n \in \mathbb{N}$, and $\Gamma$ denotes the gamma function. The fractional-order derivative is represented in the Laplace domain with zero initial conditions as

$$
L\left[{ }_{0} D_{t}^{\alpha} f(t)\right]=s^{\alpha} F(s)
$$

where $s^{\alpha}$ is a fractional Laplacian operator. The fractional R-L integration of an arbitrary function $f(t)$ is given by

$$
\left(I_{a}^{\alpha} f\right)(t)=\frac{1}{\Gamma(\alpha)} \int_{a}^{t} \frac{f(\tau)}{(t-\tau)^{1-\alpha}} \mathrm{d} \tau .
$$

By using the convolution property, (46) can be further simplified as

$$
\left(I_{a}^{\alpha} f\right)(t)=\frac{1}{\Gamma(\alpha)} t^{\alpha-1} * f(t)
$$

where $t>a$ and $*$ denotes convolution. It can be written in the Laplace domain with zero initial conditions as

$$
L\left[I_{0}^{\alpha} f(t)\right]=\frac{1}{s^{\alpha}} F(s)
$$

\section{REFERENCES}

[1] J. Wang, Y. Wei, T. Liu, A. Li, and Y. Wang, "Fully parametric identification for continuous time fractional order Hammerstein systems," J. Franklin I., 2019.

[2] Y. Zhao, Y. Li, F. Zhou, Z. Zhou, and Y. Chen, "An iterative learning approach to identify fractional order KiBaM model," IEEE/CAAJ. Autom. Sinica, vol.4, no. 2, pp. 322-331, 2017.

[3] S. W. Sung, "System identification method for Hammerstein processes," Industrial \& Engineering Chemistry Research, vol.41, no. 17, pp. 4295-4302, 2002.

[4] Y. J. Lee, S. W. Sung, S. Park, and S. Park, "Input test signal design and parameter estimation method for the Hammerstein-Wiener processes," Industrial \& Engineering Chemistry Research, vol.43, no.23, pp. 7521-7530, 2004.

[5] H. C. Park, S. W. Sung, and J. Lee, "Modeling of Hammerstein-Wiener processes with special input test signals," Industrial \& Engineering Chemistry Research, vol. 45, no. 3, pp. 1029-1038, 2006.

[6] J.-C. Jeng, M.-W. Lee, and H.-P. Huang, "Identification of blockoriented nonlinear processes using designed relay feedback tests," Industrial \& Engineering Chemistry Research, vol.44, no.7, pp. 2145-2155, 2005. 
[7] U. Mehta and S. Majhi, "Identification of a class of Wiener and Hammerstein-type nonlinear processes with monotonic static gains," ISA Trans., vol. 49, no. 4, pp. 501-509, 2010.

[8] S. Dong, T. Liu, and Q. Wang, "Identification of Hammerstein systems with time delay under load disturbance," IET Control Theory Applications, vol. 12, no. 7, pp.942-952, 2018.

[9] S. Zhang, D. Wang, and F. Liu, "Separate block-based parameter estimation method for hammerstein systems," Royal Society Open Science, vol. 5, no. 6, 2018.

[10] F. Li and L. Jia, "Parameter estimation of Hammerstein-Wiener nonlinear system with noise using special test signals," Neurocomputing, vol.344, pp.37-48, 2019.

[11] M. Aoun, R. Malti, O. Cois, and A. Oustaloup, "System identification using fractional Hammerstein models," in Proc. 15th IFAC World Congr., Spain, pp. 264-268, 2002.

[12] K. Hsu, K. Poolla, and T. L. Vincent, "Identification of structured nonlinear systems," IEEE Trans. Automatic Control, vol.53, no. 11, pp. 2497-2513, 2008.

[13] A. Maachou, R. Malti, P. Melchior, J.-L. Battaglia, A. Oustaloup, and B. Hay, "Nonlinear thermal system identification using fractional Volterra series," Control Engineering Practice, vol. 29, pp. 50-60, 2014.

[14] L. Vanbeylen, "A fractional approach to identify Wiener-Hammerstein systems," Automatica, vol.50, no. 3, pp.903-909, 2014.

[15] G. Giordano and J. Sjoberg, "A time-domain fractional approach for Wiener-Hammerstein systems identification," IFAC-PapersOnLine, vol. 48, no. 28, pp. 1232-1237, 2015.

[16] W. Allafi, I. Zajic, K. Uddin, and K. J. Burnham, "Parameter estimation of the fractional-order Hammerstein-Wiener model using simplified refined instrumental variable fractional-order continuous time," IET Control Theory Applications, vol. 11, no. 15, pp. 2591-2598, 2017.

[17] V. S. Krishnasamy, S. Mashayekhi, and M. Razzaghi, "Numerical solutions of fractional differential equations by using fractional Taylor basis," IEEE/CAA J. Autom. Sinica, vol. 4, no. 1, pp. 98-106, 2017.

[18] W. Gu, Y. Yu, and W. Hu, "Artificial bee colony algorithmbased parameter estimation of fractional-order chaotic system with time delay," IEEE/CAA J. Autom. Sinica, vol. 4, no. 1, pp. 107-113, 2017.

[19] N. I. Chaudhary, M. S. Aslam, and M. A. Z. Raja, "Modified volterra $1 \mathrm{~ms}$ algorithm to fractional order for identification of Hammerstein nonlinear system," IET Signal Processing, vol.11, no.8, pp.975-985, 2017.

[20] D. Cai, Y. Yu, and J. Wei, "A modified artificial Bee colony algorithm for parameter estimation of fractional-order nonlinear systems," IEEE Access, vol. 6, pp.48600-48610, 2018.

[21] M. J. Moghaddam, H. Mojallali, and M. Teshnehlab, "Recursive identification of multiple-input single-output fractional-order Hammerstein model with time delay," Applied Soft Computing, vol. 70, pp. 486-500, 2018.

[22] S. Cheng, Y. Wei, D. Sheng, Y. Chen, and Y. Wang, "Identification for Hammerstein nonlinear ARMAX systems based on multi-innovation fractional order stochastic gradient," Signal Processing, vol.142, pp. 1-10, 2018.

[23] M.-R. Rahmani and M. Farrokhi, "Fractional-order Hammerstein statespace modeling of nonlinear dynamic systems from input-output measurements," ISA Trans., 2019.

[24] Z. Aslipour and A. Yazdizadeh, "Identification of nonlinear systems using adaptive variable-order fractional neural networks (case study: a wind turbine with practical results)," Engineering Applications of Artificial Intelligence, vol. 85, pp.462-473, 2019.

[25] S. Cheng, Y. Wei, D. Sheng, and Y. Wang, "Identification for Hammerstein nonlinear systems based on universal spline fractional order LMS algorithm," Communications in Nonlinear Science and Numerical Simulation, vol. 79, pp. 104901, 2019.

[26] Y. Li, X. Meng, and Y.-Q. Ding, "Using wavelet multi-resolution nature to accelerate the identification of fractional order system," Chinese Physics B, vol.26, no. 5, pp. 050201, 2017.

[27] Y. Li, X. Meng, B. Zheng, and Y. Ding, "Parameter identification of fractional order linear system based on Haar wavelet operational matrix," ISA Trans., vol. 59, pp. 79-84, 2015.

[28] Y. Tang, N. Li, M. Liu, Y. Lu, and W. Wang, "Identification of fractionalorder systems with time delays using block pulse functions,' Mechanical Systems and Signal Processing, vol.91, pp.382-394, 2017.

[29] K. Kothari, U. Mehta, and J. Vanualailai, "A novel approach of fractional-order time delay system modeling based on Haar wavelet,' ISA Trans., vol. 80, pp.371-380, 2018.

[30] X.-L. Luo, L.-Z. Liao, and H. W. Tam, "Convergence analysis of the Levenberg-Marquardt method," Optimization Methods and Software, vol. 22, no. 4, pp.659-678, 2007.

[31] M. A. Demetriou and I. G. Rosen, "On the persistence of excitation in the adaptive estimation of distributed parameter systems," IEEE Trans. Automatic Control, vol.39, no. 5, pp. 1117-1123, 1994.

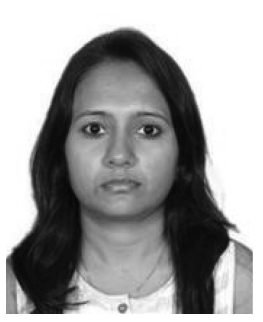

Kajal Kothari received her M.Tech degree in Communication Systems from Sardar Vallabhbhai $\mathrm{Na}$ tional Institute of Technology, India. Currently, she is a $\mathrm{PhD}$ student at the School of Engineering and Physics, The University of the South Pacific, Fiji. Her research interests include image processing, system modeling and identification, fractional systems and control.

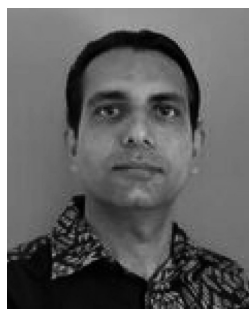

tion.
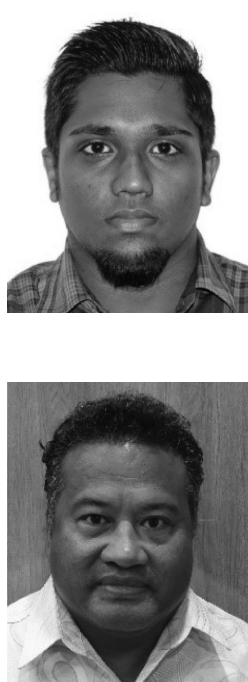

Utkal Mehta is a Senior IEEE Member (SM'15) and obtained his $\mathrm{PhD}$ from IIT Guwahati, India, in the area of system identification and process control. He is now working in Electrical and Electronics Engineering from the University of the South Pacific, Fiji as Associate Professor. His current research focuses on process identification, applied fractional calculus (AFC) for modeling, fractional-order filter design on reconfigurable devices like FPAA and various robotics applications for medical and industrial automa-

Vineet Prasad received his B.E degree in Electrical and Electronics engineering from the University of the South Pacific, Fiji, in 2018. He is currently pursuing his M.Sc. degree at the University of the South Pacific. His current research interests include fractional-order systems theory, non-linear system modelling and identification, and control systems.

Jito Vanualailai obtained his $\mathrm{PhD}$ from Kobe University, Japan, in 1994, in the area of stability analysis of nonlinear systems. He joined the University of the South Pacific, Fiji, thereafter, as Lecturer in Mathematics. He is now Professor of Applied Mathematics and the Director of Research at the University of the South Pacific. His research interests include Stability of Nonlinear Systems, Artificial Neural Networks, Volterra Integro-differential Systems, Planning Algorithms, and Swarm Intelligence. 